\title{
Melting of Colloidal Molecular Crystals on Triangular Lattices
}

\author{
A. Šarlah, ${ }^{1, *}$ T. Franosch, ${ }^{1,2}$ and E. Frey ${ }^{2,1}$ \\ ${ }^{1}$ Hahn-Meitner-Institut, Abteilung Theorie, Glienicker Strasse 100, D-14109 Berlin, Germany \\ ${ }^{2}$ Arnold Sommerfeld Center and CeNS, Department of Physics, Ludwig-Maximilians-Universität München,
} Theresienstrasse 37, D-80333 München, Germany

(Received 6 August 2004; published 17 August 2005)

\begin{abstract}
The phase behavior of a two-dimensional colloidal system subject to a commensurate triangular potential is investigated. We consider the integer number of colloids in each potential minimum as rigid composite objects with effective discrete degrees of freedom. It is shown that there is a rich variety of phases including "herringbone" and "Japanese 6 in 1" phases. The ensuing phase diagram and phase transitions are analyzed analytically within variational mean-field theory and supplemented by Monte Carlo simulations. Consequences for experiments are discussed.
\end{abstract}

DOI: 10.1103/PhysRevLett.95.088302

PACS numbers: 82.70.Dd, 64.70.Dv

In the past decade it has been realized that soft materials can serve as versatile model systems to study phenomena of condensed matter physics. In particular, twodimensional (2D) systems of colloidal particles interacting with light sources are ideal to mimic the adsorption of atoms and molecules on atomic surfaces, vortices in superconductors with periodic pinning arrays, and many other related phenomena [1-3]. Experimental studies on flat substrates [4] have beautifully confirmed the existence of a two-stage melting process mediated by the successive unbinding of dislocations [5] and disclinations [6]. If confined to a 1D periodic potential, 2D colloidal systems show even richer behavior with reentrant melting [7] and novel phases such as the locked floating solid [8,9]. Results from analytical theories have been complemented by a series of numerical simulations (see, e.g., Refs. [10-12]).

Recent experimental $[13,14]$ and theoretical $[15,16]$ investigations have studied the effect of $2 D$ periodic potentials on the phase behavior. In particular, experiments on triangular light lattices [13] with a stoichiometry of three colloids per site have motivated our research. One observes that even at rather low potential strength the colloids tend to group as trimers forming an almost equilateral triangle, but with a quite significant number of defects, i.e., groups of two or four particles. Upon increasing the potential strength, the number of defects decreases rapidly such that they become unobservable already for moderate intensities. Then, the important low energy excitations are the orientation of the trimers, regarded as rigid composite objects, with respect to the lattice direction. Because of the interaction of the trimers, long-range orientational order is expected for sufficiently strong coupling. Alignment of the trimers is observed experimentally [13] as soon as the defect-free regime takes over. Interestingly, the same authors also observed a loss of orientational long-range order at even higher intensities, which was interpreted as reentrant melting and appears to be confirmed by computer simulations [15].
In this Letter we derive phase diagrams for commensurate colloidal systems in high external fields by analytic methods supplemented by Monte Carlo (MC) simulations. The key idea is to reduce the problem to the low energy degrees of freedom by considering the integer number of colloidal particles that gather in a single potential minimum as a rigid composite object. Its shape is determined by the symmetry of the lattice and the number of the constituting colloidal particles, and its size by the interplay of interparticle repulsion and external potential. Short-time orientational fluctuations close to the potential minima are considered to be already averaged out, leaving only a discrete set of gross orientations. We mainly focus on dimers on a triangular lattice since they exhibit a rich phase diagram and exhibit a series of intriguing phases. At the end of the Letter we report how the phase diagram for trimers can be obtained in a rather straightforward manner.

For an isolated dimer there are three equivalent orientational ground states on a triangular lattice, denoted by $\sigma=$ 1, 2, 3 (see Fig. 1). The effective interaction between the dimers results from the screened Coulomb interaction between the constituent colloids, which is short ranged for the experimental conditions in Ref. [13]. Therefore, it is appropriate to restrict the effective dimer-dimer interaction to nearest neighbors. A pair of dimers can be in $3^{2}$

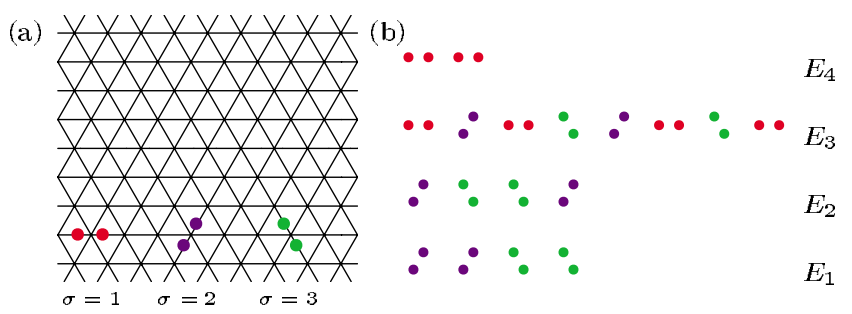

FIG. 1 (color online). The model system. (a) The triangular lattice of the external field and the three orientational states of the dimers. (b) Four energy levels for the pairwise interactions of dimers. 
configurations corresponding to one of four energy levels, with generic ordering $E_{1}<E_{2}<E_{3}<E_{4}$; see Fig. 1 . The relation between these model parameters and the experimental control parameters like screening length and potential strength can be worked out in detail [17], but is far too involved to be presented here. As a guidance there are the following trends. Increasing the laser potential compresses the dimers and thus by increasing the effective distances between colloids of neighboring dimers reduces their interaction energies and hence the values of $E_{i}$ and the spacings between them. This is the basic mechanism underlying orientational melting. Lowering the screening length effectively corresponds to increasing the potential strength.

The Hamiltonian of our model system reads

$$
\mathcal{H}=\sum_{\alpha=1}^{3} \sum_{\langle i, j\rangle_{\alpha}} \underline{\sigma}_{i}^{\top} \cdot \mathrm{H}_{\alpha} \cdot \underline{\sigma}_{j},
$$

where $\langle i, j\rangle_{\alpha}$ denotes the pair of nearest neighbors whose bond vector is parallel to the orientation of the dimer state $\alpha . \underline{\sigma}_{i}$ is a vector representation of the dimer configuration $\sigma_{i}$ at the site $i: 1 \mapsto \underline{\sigma}=(1,0,0), 2 \mapsto \underline{\sigma}=(0,1,0)$, and $3 \mapsto \underline{\sigma}=(0,0,1)$. For the energy matrices $\mathrm{H}_{\alpha}$ one finds in terms of a permutation matrix

$$
\begin{aligned}
& \mathrm{H}_{1}=\left(\begin{array}{lll}
E_{4} & E_{3} & E_{3} \\
E_{3} & E_{1} & E_{2} \\
E_{3} & E_{2} & E_{1}
\end{array}\right), \quad \mathrm{P}=\left(\begin{array}{ccc}
0 & 1 & 0 \\
0 & 0 & 1 \\
1 & 0 & 0
\end{array}\right), \\
& \mathrm{H}_{2}=\mathrm{P}^{\top} \mathrm{H}_{1} \mathrm{P}, \quad \mathrm{H}_{3}=\mathrm{PH}_{1} \mathrm{P}^{\top} .
\end{aligned}
$$

Since a shift of the global energy scale does not affect the phase behavior, only the three energy differences in units of $k_{B} T$ constitute dimensionless parameters. Thus one would be led to expect a three-dimensional phase diagram. However, upon rewriting the Hamiltonian in "spin language" it turns out that the parameter space can actually be reduced to only two dimensions [17]. We find, omitting an additive constant,

$$
\mathcal{H}=-K \sum_{\langle i, j\rangle} \delta_{\sigma_{i}, \sigma_{j}}-M \sum_{\alpha=1}^{3} \sum_{\langle i, j\rangle_{\alpha}} \delta_{\sigma_{i}, \alpha} \delta_{\sigma_{j}, \alpha},
$$

where the new energy scales $K, M$ are given in terms of the direct dimer interaction energies by $K=-E_{1}+E_{2}$ and $M=E_{1}-2 E_{2}+2 E_{3}-E_{4}$. This reduction greatly simplifies the analysis and facilitates comparison with future experiments with the two generic control parameters, screening length and laser intensity [18].

For $M=0$ the Hamiltonian reduces to a 3-state Potts model. The critical properties for the 2D $q$-state Potts model for $q \geq 4$ can be determined exactly for ferromagnetic (FM) interactions, $K>0$. The critical point is known rigorously for square, triangular, and honeycomb lattices $[19,20]$. There is sufficient numerical evidence that the $q \geq$ 4 solution also holds for $q=3$ although an analytic proof is still missing. The FM-P (paramagnetic) phase transition of the 3-state Potts model is continuous as suggested by the assumed "exact" solution and corroborated by MC simulations and renormalization group analysis [20]. Interestingly, a mean-field description gives qualitatively wrong results, since it predicts a discontinuous transition. For negative exchange coupling $K<0$ the low temperature phase is an antiferromagnetic (AM) Potts state. The results of renormalization group, series expansions, and MC simulations for the transition on a triangular lattice appear to be controversial as far as the nature of the transition is concerned [20].

The phase diagram of dimers in the external potential forming a triangular lattice as prescribed by a generalized Hamiltonian, Eq. (3), has a richer topology. Generically $K \neq 0$, and one distinguishes between $K>0$ and $K<0$. In both cases there are two dimensionless parameters, the ratio of the exchange couplings $\tilde{M}=M /|K|$ and the normalized temperature $\tilde{T}=k_{B} T /|K|$.

An exact analytic solution of the whole phase diagram, except for the two Potts points, is difficult to obtain, and one has to rely on approximative and/or numerical methods. We start with a mean-field (MF) analysis, which allows us to determine the topology of the phase diagram and thereby the symmetry of the order parameter in the various low temperature phases. In particular, we employ a variational MF approach where the full density matrix is approximated as a product of single-site density matrices, $\rho\left(\left\{\sigma_{i}\right\}\right)=\prod_{i} \rho_{i}\left(\sigma_{i}\right)$. By choosing site-independent density matrices the order parameter of the FM phase has been found by minimizing the variational free energy. Appropriate generalizations have been introduced to obtain AM phases and other ordered phases; details will be presented elsewhere [17].

From the MF analysis we find the equation of state for the respective order parameters and, in particular, the phase boundaries. It turns out that the variational free energy for colloidal dimers in the P-FM or AM phase is identical to the corresponding result for the Potts model, provided one substitutes the Potts exchange energy $K$ by the effective

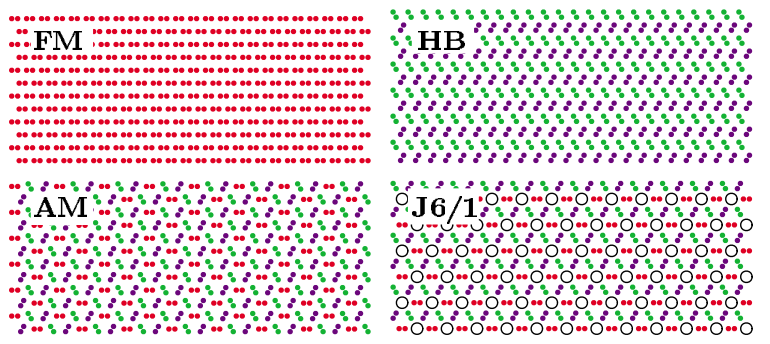

FIG. 2 (color online). Zero temperature ordered structures of 2D colloidal dimers: (a) ferromagnetic (FM), (b) herringbone (HB), (c) Potts antiferromagnetic (AM), and (d) Japanese 6 in 1 $(\mathrm{J} 6 / 1)$, structure. In the latter, the energy of the system does not depend on the orientation of dimers on sites denoted by circles; thus this structure is $3^{N / 4}$ degenerate. 
coupling $K \rightarrow K_{\text {eff }}=K+M / 3$. The critical temperatures for the P-FM and P-AM phase transition are located at $k_{B} T_{c}=3 K_{\text {eff }} /(2 \ln 2) \quad$ and $\quad k_{B} T_{c}=-3 K_{\text {eff }} /(4 \ln 2)$, respectively.

The colloidal dimer Hamiltonian, Eq. (3), allows for new intriguing structures that are not realized within the Potts model. We have investigated for herringbone (HB) and Japanese 6 in $1(\mathrm{~J} 6 / 1)$ structures, a term borrowed from weaving patterns for chainmailles; see Fig. 2. The corresponding order parameters can be obtained by (numerically) minimizing the variational free energies, and the phase boundaries can be determined accordingly; see Fig. 3. Except for the HB-P transition for $\tilde{M}<-7 / 3$ within MF theory all phase transitions are discontinuous. The HB-P transition has already been studied in the context of $\mathrm{N}_{2}$ absorbates on graphite. A continuous anisotropic planar rotor model analogous to our discrete version for $K=0$ yielded similar results within both MF and MC descriptions [21,22].

In our case, the MF description is not expected to predict the correct order of the transition, as can be inferred by specializing to the Potts model $(M=0)$. Nevertheless, the MF results should constitute reasonable approximations for the actual phase behavior. In order to gain further insight we have performed extensive MC simulations of the colloidal dimer Hamiltonian, Eq. (3), using a standard Metropolis procedure. The observables have been averaged over $\geq 5000$ statistically independent configurations after the system has been equilibrated. Every $1000 \mathrm{MC}$ cycles a new configuration contributing to the averages is considered. Simulations have been performed on several system sizes, $N=L \times L$ and $L=12,36,54,108$, and periodic boundary conditions have been employed to
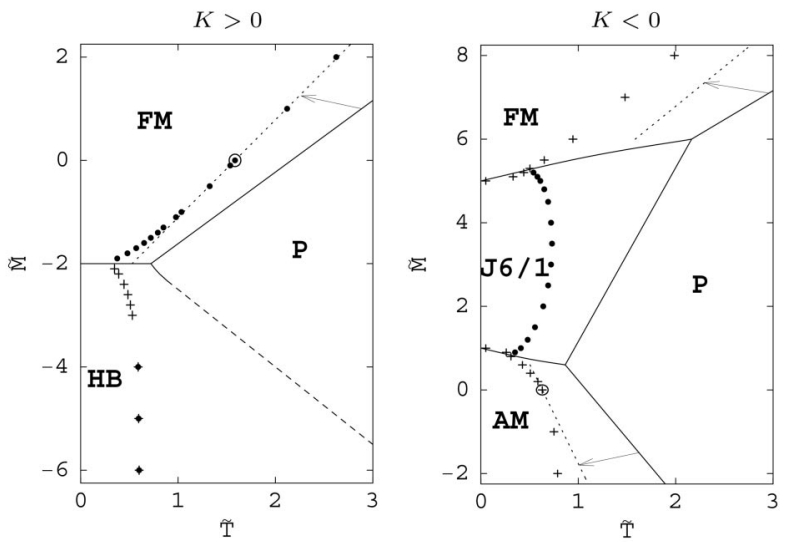

FIG. 3. Phase diagram for dimers. Solid and dashed lines represent discontinuous and continuous MF transitions. Dots and crosses denote the continuous and discontinuous transition points as obtained by MC simulations; the two encircled symbols indicate the pure Potts transitions. The nature of the HB-P transition for $\tilde{M}<-4$ is not clear, which is indicated by a dot-cross symbol for the critical point. Dotted lines represent extrapolations of the Potts model critical point; see text. mimic bulklike conditions [17]. The order parameter characterizing the various phases has been chosen in analogy with the Potts model, either defined on the whole system or suitable sublattices,

$$
S=\frac{1}{N}\left[N_{\Sigma}-\frac{1}{2} \sum_{\sigma \neq \Sigma} N_{\sigma}\right]
$$

Here, $N_{\sigma}$ is the number of dimers in state $\sigma$; in particular, $N_{\Sigma}$ corresponds to majority orientation, and $N=\Sigma_{\sigma} N_{\sigma}$ is the total number of the dimers on the whole (sub)lattice. We have performed temperature sweeps for $K>0$ and $K<0$ with a number of choices for $\tilde{M}$ starting from the ordered structures. We have monitored the average order parameter and energy, as well as their respective variance. The transition temperatures corresponding to melting of the ordered structures have been determined by extrapolating the location of the maxima of the heat capacity and susceptibility to the infinite system size. In order to decide whether the respective phase transition is strongly discontinuous or compatible with a continuous-weakly discontinuous transition scenario, we have evaluated Binder's cumulant and respective probability distributions for the order parameter and energy [17]; both scenarios are exemplified in Fig. 4.

Figure 3 exhibits the phase diagram for the colloidal dimer Hamiltonian; boundaries resulting from both MF description and $\mathrm{MC}$ simulations are included in the figure. The variational MF free energy reproduces the topology of the phase diagram, although some of the transitions become continuous by fluctuation effects. Quantitatively, MF overestimates the transition temperatures with respect to simulation results by a factor of order unity, a feature already familiar from the Ising model [19]. Interestingly, the discontinuous HB-P transition appears to saturate for large negative values of $\tilde{M}$ in strong contrast to the corresponding MF result [23]. For colloidal systems the regime corresponding to $K>0$ and $\tilde{M}<0$ should be experimentally accessible. A stringent test of our theory would be to experimentally verify the predicted HB-P phase transition.

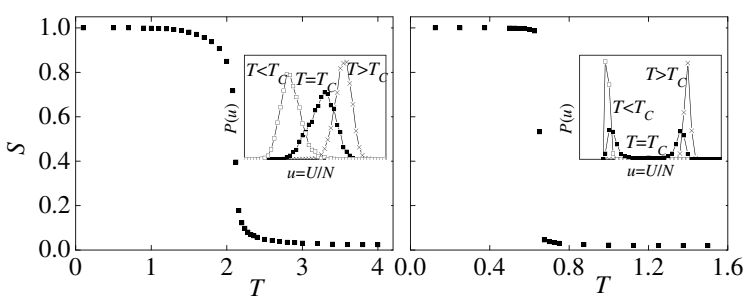

FIG. 4. Order parameter $S$ as a function of the reduced temperature for the two FM-P transitions. Left: Potts-like continuous transition for $K, M>0$. Right: the $\tilde{M}$-term driven first order transition for $K<0, \tilde{M}>5$. Inset: probability distributions of states with respect to the energy per dimer, $u=U / N$, below, above, and at the transition temperature. 
Fluctuation effects reduce the critical temperature of the Potts model by a known factor [20]. The variational MF free energy of the colloidal dimer Hamiltonian for P-FM and AM structures is equivalent to the MF Potts model, provided that the Potts exchange energy is replaced by the effective coupling $K_{\text {eff }}$. This analogy suggests an empirical improvement of the MF result for these transitions by rescaling the transition temperatures with the same respective correction factors. The result of this procedure is shown as dashed lines in Fig. 3. For $K>0$ there is excellent agreement with simulation results for temperatures $\tilde{T}>1$. For $K<0$ the mapping gives an overall quantitative improvement of the phase diagram. In particular, for $M=$ 0 the well known Potts solution is recovered.

We close with reporting our main results for trimers on a triangular lattice. Because of the symmetry of the lattice, trimers can be in only one of two orientational states, represented by Ising spins $\sigma= \pm$. Hence the four configurational states for trimer pairs contribute one out of the three possible interaction energies,

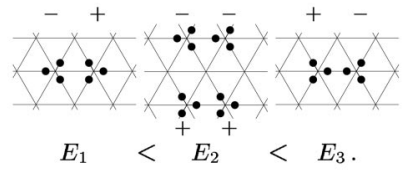

In contrast to the Ising model, the interaction energies for the $(+-)$ and $(-+)$ configurations are different. The relation between the model parameters and the control parameters of the experiments are similar as for dimers. In particular, an increase of the potential strength again leads to a reduction of the interaction energies between trimers and thus favors orientational melting.

The central questions now are how many relevant energies determine the phase behavior and what is the effective statistical model. Similar considerations as for dimers would suggest that there are two relevant energy scales resulting in a two-dimensional phase diagram. Actually, by a simple rearrangement the Hamiltonian can be cast in the form of an Ising model with a single excitation energy $\Delta E=E_{1}+E_{3}-2 E_{2}$ [17] corresponding to single trimer flips, irrespective of the configurations of the neighboring trimers. Depending on the sign of $\Delta E$, the ground state is either "ferromagnetic" with parallel $(++)$ [or equivalently $(--)$ ] configurations or "antiferromagnetic" with an alternating arrangement of $(-+)$ and $(+-)$ configurations. It is interesting to note that it is the relative orientation of trimer pairs and not the trimers themselves that determine the ground state.

For the remainder of the analysis known exact results of the 2D Ising model [19] can now be employed to obtain the phase diagram. Considering the parameters of the experimental setup in Ref. [13], we can identify the critical laser intensity, $V_{0}=78 k_{B} T$ [17], above which the orientational order of trimers is lost. This result is consistent with experimental observations, where the colloidal trimer system was found to be orientationally ordered for $V_{0}=$ $60 k_{B} T$ but disordered for $V_{0}=110 k_{B} T$. It would be interesting to extend the experimental analysis to find the actual transition point and compare it with our prediction.

We thank C. Bechinger for discussions. A. $\breve{S}$. acknowledges the support by the Humboldt Foundation.

*On leave from University of Ljubljana, Ljubljana, Slovenia.

[1] A. J. Berlinsky and A. B. Harris, Phys. Rev. Lett. 40, 1579 (1978).

[2] V.L. Pokrovsky, Theory of Incommensurate Crystals (Harwood, New York, 1984).

[3] T. Matsuda et al., Science 271, 1393 (1996).

[4] C. A. Murray and D. H. V. Winkle, Phys. Rev. Lett. 58, 1200 (1987).

[5] J. M. Kosterlitz and D. J. Thouless, J. Phys. C 6, 1181 (1973).

[6] D. R. Nelson and B. I. Halperin, Phys. Rev. B 19, 2457 (1979).

[7] Q.-H. Wei, C. Bechinger, D. Rudhardt, and P. Leiderer, Phys. Rev. Lett. 81, 2606 (1998).

[8] E. Frey, D. R. Nelson, and L. Radzihovsky, Phys. Rev. Lett. 83, 2977 (1999).

[9] J. Baumgartl, M. Brunner, and C. Bechinger, Phys. Rev. Lett. 93, 168301 (2004).

[10] K. Loudiyi and B. J. Ackerson, Physica (Amsterdam) 184A, 26 (1992).

[11] J. Chakrabarti et al., Phys. Rev. Lett. 75, 2232 (1995); C. Das et al., Curr. Sci. 80, 959 (2001).

[12] W. Strepp, S. Sengupta, and P. Nielaba, Phys. Rev. E 66, 056109 (2002).

[13] M. Brunner and C. Bechinger, Phys. Rev. Lett. 88, 248302 (2002).

[14] K. Mangold, P. Leiderer, and C. Bechinger, Phys. Rev. Lett. 90, 158302 (2003).

[15] C. Reichhardt and C. J. Olson, Phys. Rev. Lett. 88, 248301 (2002).

[16] R. Agra, F. van Wijland, and E. Trizac, Phys. Rev. Lett. 93, 018304 (2004).

[17] A. Šarlah, T. Franosch, and E. Frey (unpublished).

[18] Upon increasing both the potential strength and the screening length the ratio $|M| / K$ becomes smaller.

[19] R.J. Baxter, Exactly Solved Models in Statistical Mechanics (Academic Press, London, 1989).

[20] F. Y. Wu, Rev. Mod. Phys. 54, 235 (1982).

[21] O. G. Mouritsen and A. J. Berlinsky, Phys. Rev. Lett. 48, 181 (1982).

[22] D. Marx et al., Mol. Phys. 83, 31 (1994).

[23] Single-dimer flips of the HB ground state correspond to excitation energies $2 K$ and $2|M|$ depending on the two possible new orientations of the dimer. For $-M \gg K$, $M$-type excitations are strongly suppressed. However, the MF approach, in which generically the role of $M$ is to renormalize the energy $K$ in a linear way, misses that. 